\title{
Subsurface imaging of two-dimensional materials on the nanoscale
}

Franco Dinelli $^{\rho}$, Pasqualantonio Pingue ${ }^{\theta}$, Nicholas D. Kay ${ }^{\dagger}$ and Oleg V. Kolosov ${ }^{\dagger}$

$\rho$ CNR, Istituto Nazionale di Ottica (INO), via Moruzzi 1, 56124 Pisa, Italy

$\theta$ Laboratorio NEST - Scuola Normale Superiore, and Istituto Nanoscienze - CNR, Piazza San

Silvestro 12, I-56127 Pisa, Italy

$\dagger$ Physics Department, Lancaster University, Lancaster, LA1 4YB, UK

\begin{abstract}
Scanning probe Microscopy (SPM) represents a powerful tool that, in the past thirty years, has allowed one to investigate material surfaces in unprecedented ways at the nanoscale level. However, SPM has shown very little power of depth penetration, whereas several nanotechnology applications would require it. Subsurface imaging has been achieved only in a few cases, when subsurface features influence the physical properties of the surface, such as the electronic states or the heat transfer. Ultrasonic Force Microscopy (UFM), an adaption of the Atomic Force Microscopy (AFM) contact mode, can dynamically measure the stiffness of the elastic contact between the probing tip and the sample surface. In particular, UFM has proven highly sensitive to the near-surface elastic field in non-homogeneous samples.

In this paper, we present an investigation of two-dimensional (2D) materials, namely flakes of graphite and molybdenum disulphide placed on structured polymeric substrates. We show that UFM can non-destructively distinguish suspended and supported areas and localize defects, such as buckling or delamination of adjacent monolayers, generated by residual stress. Specifically, UFM can probe small variations in the local indentation induced by the mechanical interaction between tip and sample. Therefore, any change in the elastic modulus within the volume perturbed by the applied load or the flexural bending of the suspended areas can be detected and imaged. Such a power of investigation is very promising in order to investigate the buried interfaces of nanostructured 2D materials such as in graphene-based devices.
\end{abstract}

\section{Keywords:}

SPM, AFM, UFM, 2D Materials, Graphene, MoS2, Elastic Properties, Subsurface. 


\section{Introduction}

In the past decades Scanning Probe Microscopy (SPM) has represented a major breakthrough in surface science, allowing investigations on the nanoscale not even thinkable before. ${ }^{1,2}$ The range of studies is so vast that any attempt to review it would fall short. ${ }^{3,4}$ Nevertheless, the same near-field principles, that allow SPM to achieve high spatial resolution, inevitably confine the investigations to the surface or at best to shallow regions a few angstroms deep. On the other hand, subsurface detection is more and more required due to the ever-growing development of new nanotechnology applications, namely, multilayer high integration chips, ultrathin coatings or confined semiconductor hetero-structures. For instance, it would be extremely important to characterize buried interfaces, such as in graphene-based devices. ${ }^{5}$

Examples of subsurface detection with nanoscale resolution are so far very few. The literature reports of some operational modes sensitive to the electronic states or thermal properties. In Scanning Tunnelling Microscopy (STM), the surface electronic states can be affected by the presence of subsurface features. ${ }^{6,7,8}$ In Electric Force Microscopy (EFM), charges located below the surface can similarly influence the electrostatic force or the surface displacement sensed by the tip.9,10,11 Finally, in Scanning Thermal Microscopy (SThM), the thermal dissipation depends to a great extent on a given volume located under the tip and any non-homogeneity can be thus probed. ${ }^{12,13,14}$

A chapter on its own deserves subsurface imaging through the detection of the elastic properties of materials. Ultrasonic Force Microscopy (UFM) is a technique invented by Kolosov and Yamanaka, ${ }^{15}$ resulting from an adaption of Atomic Force Microscopy (AFM) working in Contact Mode (CM). It was initially proposed and developed in order to overcome the limits of Force Modulation Microscopy (FMM). ${ }^{16}$ FMM is indeed limited to stiffness values of the order of the cantilever spring constant, which is typically small in CM-AFM in order not to damage the sample or the tip while characterizing the morphology. A class of operational modes can be associated as stemmed directly from UFM or developed in the same period: Scanning Acoustic Force Microscopy (SAFM), ${ }^{17}$ Atomic Force Acoustic Microscopy (AFAM), ${ }^{18}$ Scanning Local Acceleration Microscopy $(\text { SLAM })^{19}$ and Heterodyne Force Microscopy (HFM). ${ }^{20}$ In AFAM, in particular, one excites and detects higher eigenmodes of the cantilever, making it more sensitive to the elastic field than FMM. A similar approach is based on a multi-frequency detection but operates in Tapping Mode $(\mathrm{TM})^{21}$ rather than in CM-AFM. ${ }^{22}$ In particular, Ebeling et al. have been able 
to image glass nanoparticles (NPs) embedded in a soft Polydimethylsiloxane (PDMS) film. 23

UFM has specifically proven a valid tool to localize subsurface defects in stiff materials. This can be achieved working at low load values and eliminating the shear stress at contact thanks to a superlubricity phenomenon ultrasonically induced. ${ }^{24}$ Yamanaka et al. have firstly reported images showing subsurface features in graphite, ${ }^{25}$ attributing them to dislocations between adjacent graphene layers. ${ }^{26}$ Afterwards, Checanov et al. have imaged cracks in hard disk heads ${ }^{27}$ and Dinelli et al. have shown other examples, such as the detachment of $\mathrm{Al}$ coatings grown on polymer films or buried rubber spheres in a Poly(methyl methacrylate) (PMMA) matrix. ${ }^{28}$ Finally, HFM has been employed to detect NPs deeply buried in polymeric matrixes. ${ }^{29}$ While the theoretical explanation of the nature of the subsurface contrast, whether it is due to ultrasonic wave scattering or elastic field modification, is still under discussion, ${ }^{30}$ recent investigations have confirmed the reproducibility of this experimental observation. ${ }^{31,32}$

Based on these premises, we have carried out a study of samples made of two-dimensional (2D) materials, ${ }^{33}$ targeting the exploration of subsurface details by means of UFM. In particular, we have deposited thin flakes of graphite and molybdenum disulphide $\left(\mathrm{MoS}_{2}\right)$ on structured polymeric substrates in order to obtain suspended and supported areas. UFM data are also compared to data obtained with TM-AFM and Peak Force modes, the latter with a Quantitative NanoMechanical (QNM) real-time software analysis. ${ }^{34}$ Our results are then discussed and interpreted according to well established models of contact mechanics.

\section{Experimental}

Graphite or $\mathrm{MoS}_{2}$ thin flakes can be transferred to a given substrate exploiting a PDMS stamp based technique. ${ }^{35}$ In our case the substrate chosen is a film of Cyclic Olefin Copolymer (COC) polymer patterned via hot embossing in order to produce periodic flat mesas with randomly distributed voids in the regular array of grooves on a macroscopic scale (around $5 \times 5 \mathrm{~mm}^{2}$ ). The height and the periodicity of the grooves are approximately $250 \mathrm{~nm}$ and $1 \mu \mathrm{m}$, respectively. Given the typical lateral size of the flakes in the range from 5 to $20 \mu \mathrm{m}$, they present adjacent regions alternatively supported by the COC mesas and suspended over the voids.

The UFM setup is based on a standard CM-AFM including a vibrating sample stage, capable of producing out-of-plane ultrasonic vibrations, with amplitude $a$, that are 
transferred to the specimen reversibly bonded to the stage with a low melting point crystalline compound, such as phenyl salicylate. ${ }^{36}$ The AFM tip and the sample surface are in contact under a fixed average load $F_{N}$, equal to the product of the cantilever stiffness $k_{\text {lever }}$ and its deflection $d_{\text {lever. }}$

At static equilibrium, in order to sustain the pressure exerts by the probe, the surface under the tip deforms by $\delta_{\text {cont }}$ that depends on the contact stiffness $k_{\text {cont }}$, defined as $\delta_{\text {cont }}=$ $F_{N} / k_{\text {cont, }}$ (see Figure $1 \mathrm{~A}$ and $\mathrm{B}$ ). The equations describing it are those of a simplified "two-spring model" (Figure 1C):

$F_{N}=k_{\text {lever }} \times d_{\text {lever }}=k_{\text {cont }} \times \delta_{\text {cont }}$

In UFM technique the excitation frequency must be much larger than the first cantilever resonance (in contact) and should not coincide with the higher resonance modes (again in contact). Working in this "inertial" regime, the cantilever cannot follow the vertical modulation and the effective cantilever spring constant $\left(k_{\text {lever }}^{\text {eff }}\right)$ results: ${ }^{37}$

$k_{\text {lever }}^{\text {eff }}>k_{\text {cont }} \approx k_{\text {lever }} \times\left(\frac{f_{U F M}}{f_{\text {Res }}}\right)^{3 / 2} \gg k_{\text {lever }}$

$k_{\text {lever }}^{\text {eff }}>k_{\text {cont }}$

where $f_{U F M}$ is the ultrasonic frequency and $f_{\text {Res }}$ the first free resonant frequency of the cantilever.

Thus, during an ultrasonic cycle, the local indentation can be modulated. In particular, as shown in Figure $1 \mathrm{~B}$, there is a critical value at which the contact breaks: i. e. $a_{1}=\delta_{1}$ corresponding to $F_{N}=F_{1}$. Beyond that, the non-linearity of the $F_{N}$ versus $\delta_{\text {cont }}$ curve produces a rectification effect that can be detected as an additional positive deflection of the cantilever. ${ }^{38}$ Modulating the ultrasonic amplitude, one can measure this critical value point by point with a lock-in amplifier while simultaneously sampling topography, lateral force or any other required signal (see Supporting Information). When the adhesion properties of the sample are homogenous, the contrast of an UFM image can be interpreted as follows: brighter colours indicate regions where the indentation is lower, corresponding to areas with larger Young's modulus. ${ }^{36}$

For the UFM and TM-AFM measurements, our experimental setups are hybrid systems made of commercial and custom components. One is based on a Multimode-type head with a Nanoscope III controller (Bruker), the other on a SMENA-type head (NT-MDT) with home-built electronic controller. Both these systems are equipped with a custom sample-holder made of a piezo-disc having a typical resonance around 2 or $4 \mathrm{MHz}$ (Physik Instrumente). For the Peak Force - QNM measurements, presented in the Supporting 
Information, an Icon-type head AFM system with a Nanoscope $V$ controller (Bruker) has been employed.

\section{Results}

In Figure 2, we show some data obtained for a graphite flake (around 50nm in thickness, excluding folded areas) deposited on a patterned COC film, described above. Figures $2 \mathrm{~A}$ and $\mathrm{B}$ show the topography and phase images obtained with standard TM-AFM. The topography image is smooth, and it is not possible to identify suspended or supported regions within the flake. Another important issue to be underlined is represented by the fact that TM-AFM phase signal is almost constant: an indication that the adhesive properties of the surface are homogeneous across this flake. The same is valid also for all the other flakes we have analysed. This fact has been confirmed through a characterization by means of QNM (see Supporting Information).

Figures $2 \mathrm{C}$ and $\mathrm{D}$ show the same flake imaged with UFM. On the one hand, the topography $(\mathrm{C})$ is identical to the topography acquired by means of TM-AFM. This is an indication that no surface damage occurs, also thanks to the superlubricity effect ultrasonically induced. ${ }^{24}$ On the other hand, the UFM contrast (D) clearly identifies the supported from the suspended portions, the latter appearing darker. In Figure 2E, the profiles of the lines drawn in Figure 2C and D are presented. The topographic profile does not show any major variation that can be attributed to the presence of voids. In the UFM profile the signal is smaller in correspondence of the voids underneath the flake with a minimum at the centre of the suspended areas. This minimum depends on the lateral size of the suspended regions. The UFM signal depends also on the flake thickness.

In Figure 3, we show a second example, represented by a MoS 2 flake (from 10 to $50 \mathrm{~nm}$ thick) placed on a COC film, patterned with parallel grooves randomly bridged. In this case, the flake is thinner than the graphite flake above reported. The topography signal faintly reveals the presence of grooves underneath, as the flake is less rigid and its shape is affected by the substrate topography. On the other hand, the UFM contrast is even higher than for the case of the graphite flake, whereas it still depends on the flake thickness and on the lateral size of the suspended regions.

In Figure 4, we focus on another specific issue already observable also in some details of the flake shown in Figure 2. For the purpose, we present two different graphite flakes placed on the same patterned COC film. Strikingly, one can observe that, besides the suspended portions, it is possible to visualize other features that can be attributed to the 
internal structure of the flake itself (see arrows). We suggest that these features are due to dislocations or other defects, as reported elsewhere. ${ }^{25}$ Signatures of this substructure can be observed both in the suspended and the supported regions. The fingerprints are represented by dark lines, variously long and orientated, or by finer details. A similar contrast can be observed on other graphite and $\mathrm{MoS}_{2}$ flakes, even when they are placed on the flat COC regions (see Supporting Information).

\section{Discussion}

Standard CM- and TM-AFM techniques are sensitive to the surface properties and it is not possible to visualize the suspended portions or any other internal defect. In particular, the contrast is dominated by the contact stiffness, typically much larger than the cantilever stiffness (Equation 2). In our case, the tip-surface contact can be described by the Derjagin-Muller-Toropov (DMT) model:39

$$
\begin{aligned}
& r=\left[\frac{3 R\left(F_{N}+F_{C}\right)}{4 E^{*}}\right]^{1 / 3} \\
& F_{N}+F_{C}=\frac{4}{3} \times E^{*} \times R^{1 / 2} \times \delta_{\text {cont }}^{3 / 2} \\
& F_{C}=2 \times \Delta \gamma \times \pi \times R \\
& k_{\text {cont }}=2 \times E^{*} \times r=\left[6 \times E^{* 2} \times R \times F_{N}\right]^{1 / 3} \\
& \frac{1}{E^{*}}=\frac{\left(1-v_{1}^{2}\right)}{E_{1}}+\frac{\left(1-v_{2}^{2}\right)}{E_{2}}
\end{aligned}
$$

where $r$ is the contact radius, $\Delta \gamma$ and $F_{C}$ respectively the work of adhesion and the adhesion force, $E_{i}$ and $v_{i}(i=1,2)$ respectively the Young's moduli and Poisson's ratios of tip and sample, $E^{*}$ the reduced Young's modulus, $R$ the tip radius. The stress field extends in a volume that is indicated in Figure $1 \mathrm{~A}$ and can reach a depth value of around $3 r .{ }^{40}$ The TM-AFM phase and QNM data indicate that, over a single flake, the adhesion can be considered homogeneous.

The ultrasonic wavelength applied to the sample is of about a few millimetres and therefore the whole surface always moves with the same amplitude (see Figure 5A) ${ }^{36}$ Therefore local variations in the ultrasonic amplitude cannot be responsible for the effects observed. The origin of the UFM subsurface imaging must be found elsewhere and specifically in its sensitivity to the indentation. Specifically, a surface in contact with a tip is not only locally indented but, if the underlying area is not supported and therefore suspended, must bend in order to sustain the external pressure. The whole system can be described by a simplified "three-spring model" (Figure 5B). At the static equilibrium we can thus write: 
$F_{N}=k_{\text {tot }} \times \delta_{\text {tot }}=k_{\text {lever }} \times d_{\text {lever }}=k_{\text {cont }} \times \delta_{\text {cont }}=k_{\text {flex }} \times \delta_{\text {flex }}$

$\delta_{\text {cont }}=d_{\text {lever }}+\frac{k_{\text {lever }}}{k_{\text {cont }}}$

$\delta_{\text {flex }}=d_{\text {lever }}+\frac{k_{\text {lever }}}{k_{\text {flex }}}$

$\delta_{\text {tot }}=\delta_{\text {cont }}+\delta_{\text {flex }}>\delta_{\text {cont }}$

$\frac{1}{k_{\text {tot }}}=\frac{1}{k_{\text {cont }}}+\frac{1}{k_{\text {flex }}}$

where $k_{f l e x}$ is the flexural stiffness elastic constant, $\delta_{f l e x}$ the flexural bending, $\delta_{\text {tot }}$ the total indentation, and $k_{\text {tot }}$ the total stiffness

Thus suspended areas present larger $\delta_{\text {tot }}$ and larger ultrasonic amplitude values are needed to break the contact. The profile in Figure $2 \mathrm{E}$ shows that, in the suspended areas, the UFM signal is not constant but varies as the contribution from the flexural bending to $\delta_{\text {tot }}$ is lower in correspondence of the borders and maximum in the middle, depending also on the lateral size of the suspended regions.

In our case, the suspended regions have a rectangular shape of various dimensions: perpendicular $(b)$ and parallel $(c)$ to the flat mesas, respectively. However, we can estimate that:

$$
\frac{\delta_{\text {flex }}}{h} \ll 1
$$

$2<\frac{b}{h}<25$

where $h$ is the flake's thickness.

Moreover, the $c / h$ ratio can be up to 100 . Given these boundary conditions, we can assume that the suspended regions behave like 'plates', stiff objects that undergo pure bending. Therefore, we can employ the formulae obtained from the theory of plates. However, simple equations for our experimental case, where the load is applied by the tip during scanning in different positions of the plate, are not available.

If one considers the simplest case with a point load at the centre, one can obtain analytic formulas that provide the flexural bending versus the lateral size, the thickness of the plates themselves and other parameters: ${ }^{41}$

$$
\begin{aligned}
& \delta_{f l e x}(\mathrm{x}, \mathrm{y})=\frac{4 F_{N}}{\pi^{4} D b c} \times \sum_{m=1,3, . .}^{\infty} \sum_{n=1,3, \ldots}^{\infty} \frac{\sin \frac{m \pi}{2} \sin \frac{n \pi}{2}}{\left[(m / b)^{2}+(n / c)^{2}\right]} \times \sin \frac{m \pi \mathrm{x}}{b} \times \sin \frac{n \pi \mathrm{y}}{c} \\
& D=E \times \frac{h^{3}}{12\left(1-\mathrm{v}^{2}\right)}
\end{aligned}
$$

where $E$ and $v$ are graphite Young's modulus and Poisson's ratio, respectively. The maximum bending for a square plate $(b=c)$ is always at the centre and is given by: 
$\delta_{\text {flex }}^{\max }=0,01159 \times F_{N} \times \frac{b^{2}}{D}$

Taking the dimensions obtained for the sample shown in Figure $2(b=c=1 \mu \mathrm{m}, h=$ $50 \mathrm{~nm}, F_{N}=10 \mathrm{nN}$ ) and assuming for the elastic parameters typical values obtained from the literature $(E=10 \mathrm{GPa}, v=0.165),{ }^{42}$ we obtain $\delta_{\text {flex }}{ }^{\max } \approx 1.1 \mathrm{~nm}$. For a DMT contact the corresponding $\delta_{\text {cont }}$ is $\approx 0.5 \mathrm{~nm}$, under the assumption of $R=20 \mathrm{~nm}$ and $F_{C}=5 \mathrm{nN}$.

With regards to the finer contrast observed in Figure $4 \mathrm{~B}$, we can state that similar features have been previously observed in graphite by means of UFM ${ }^{25,26}$ and very recently also by means of AFAM. ${ }^{43}$ They have been attributed to the presence of defects such as delamination located between adjacent graphene layers inside the flake itself (upper white arrow). These defects can modify the contact stiffness, if they are located within the volume where the elastic field applied by the tip extends to (see Figure 1A), or if the overall flexural stiffness is modified. Their presence, both in the suspended and the supported regions, suggest that they might have formed during the preparation or transfer processes. The same applies when similar features are present in flakes deposited on flat COC (see Supporting Information). We can also argue that their presence might be due to the stress induced by the PDMS-based transferring technique ${ }^{35,44}$ and therefore to the van der Waals attraction exerted from the substrate once the flake lies onto the COC structured film. The local strain is created in order to release this stress, determining the formation of defects imaged by means of UFM. Other finer features in Figure 4B are probably due to some surface damage or weakly bonded layers (lower white arrow).

In Figure 4D, another kind of nanometre scale texture appears, dark non-parallel lines (black arrow), only visible in the suspended regions of the flake. The graphite is indeed very rigid for in-plane stresses. These non-parallel lines observed in the UFM images may represent internal regions where bending stiffness rapidly drops. We may attribute this kind of features to buckling ${ }^{45,46}$ induced, while imaging, by the pressure exerted through the tip on the suspended zone of the graphite flake.

\section{Conclusions}

In this paper we have shown how Ultrasonic Force Microscopy (UFM) can detect and image subsurface features on the nanoscale in the case of two-dimensional materials, namely graphite and molybdenum disulphide. In particular, we have investigated flakes of a few tens of nanometres in thickness placed on structured polymeric substrates with suspended and supported areas. We have demonstrated that UFM can identify the different regions in a non-destructive way as is it highly sensitive to flexural bending 
induced by the elastic field applied between tip and surface. Subsurface imaging can be also achieved through revealing changes in the elastic modulus due to defects located within the volume perturbed by the elastic field. Thus UFM can localize, inside the flakes themselves, delamination and buckling defects induced by the residual stress, due to the preparation and transfer processes, or by the pressure exerted through the tip. For all these reasons, we suggest that this SPM technique is a very promising candidate for the mechanical characterization and testing of nano-devices based on two-dimensional materials where high spatial resolution may be requested.

\section{Acknowledgements}

FD acknowledges funding from the 'Short Term Mobility' programme by the CNR and Paolo Baschieri for helping in the construction of the UFM setup. PP acknowledges Sandro Meucci for COC sample preparation and the 'Graphene Flagship' initiative for supporting his stay at Lancaster University. OVK acknowledges support of the EC grant 'QUANTIHEAT' (grant agreement $n^{\circ} 604668$ ), EPSRC funding to 'Graphene NowNANO', and Lancaster University support. 

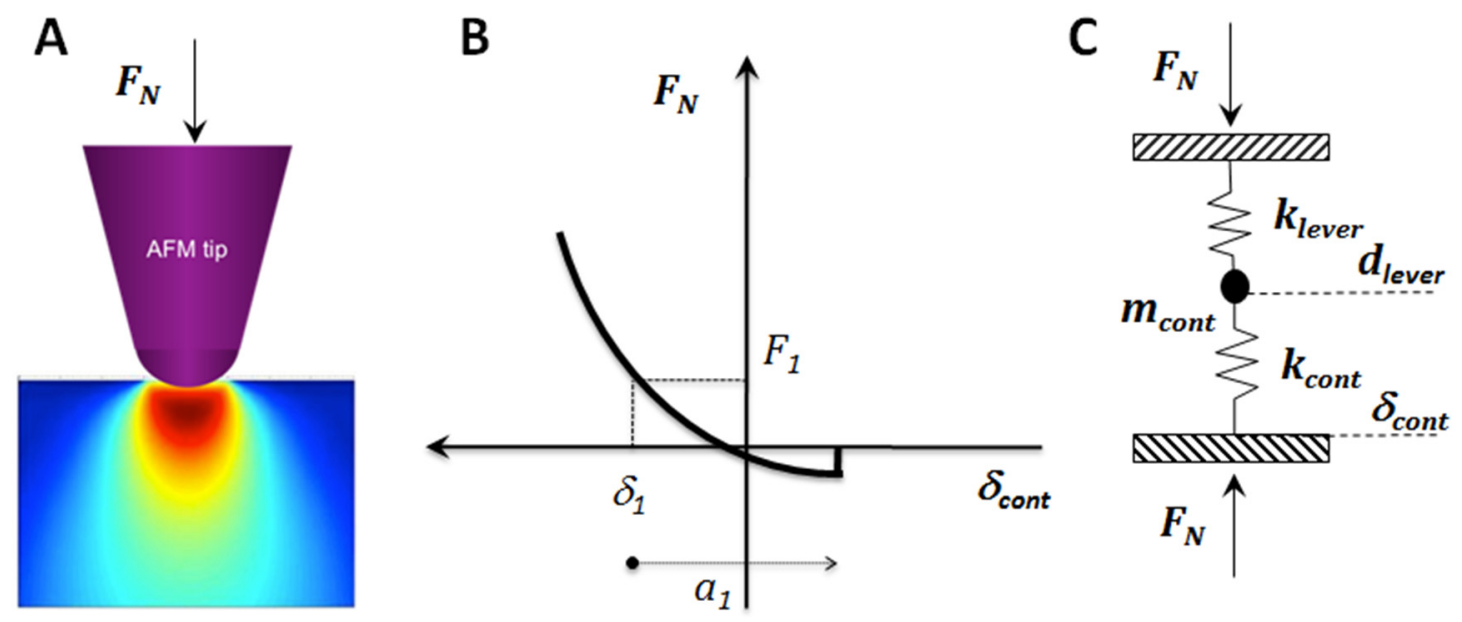

Figure 1: Schematic drawings of: $(A)$ the strain field induced by a tip in contact with a surface under a load $F_{N}$; (B) a load $F_{N}$ versus indentation $\delta$ curve; (C) a two spring model for an elastic contact, where $k_{\text {cont }}$ represents the contact stiffness and $k_{\text {lever }}$ the cantilever elastic constant. For a given load applied $F_{1}, a_{1}$ represents the minimum ultrasonic amplitude, equal to the local indentation $\delta_{1}$, at which the contact breaks. 

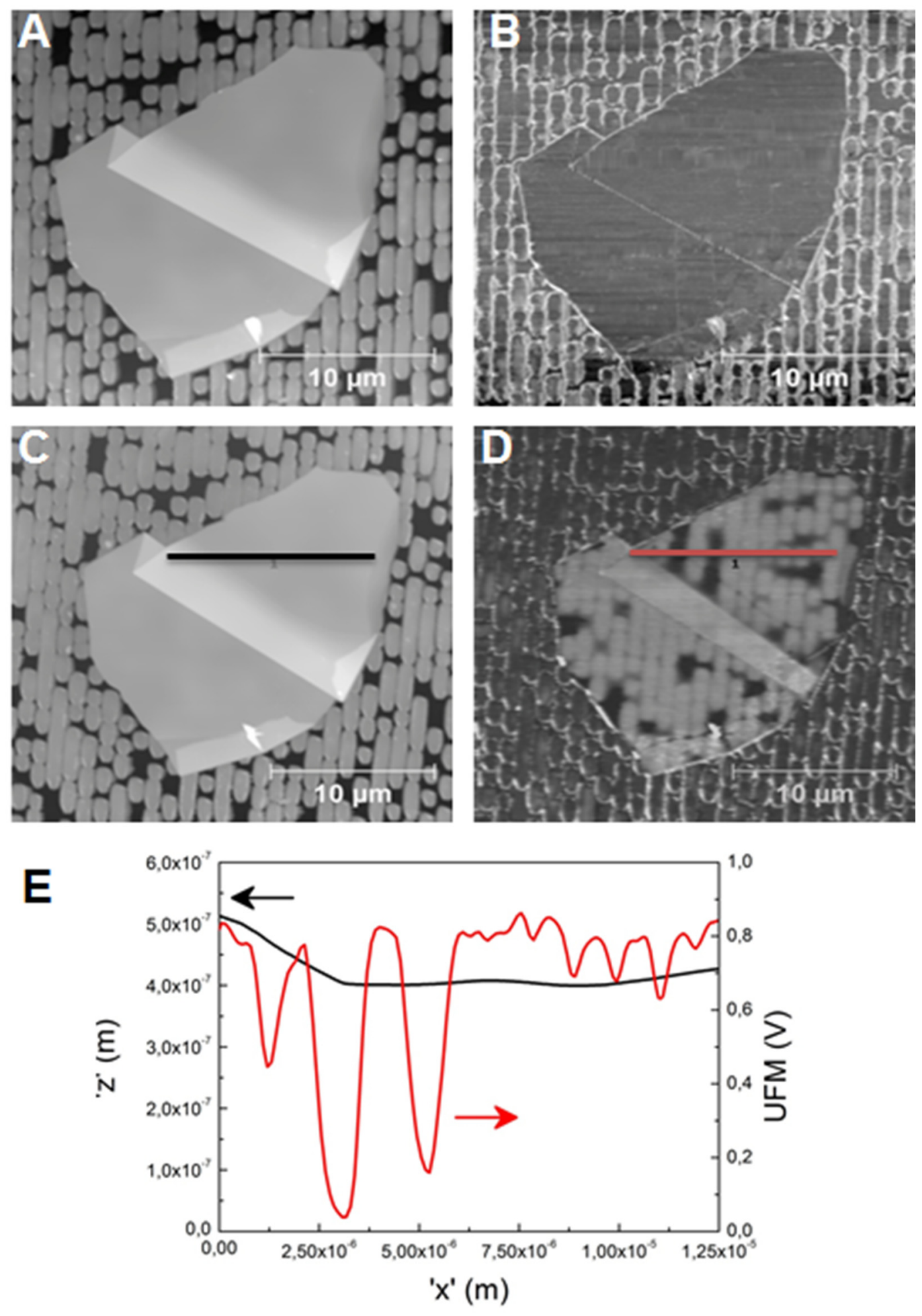

Figure 2: A graphite flake (around $50 \mathrm{~nm}$ thick, excluding folded areas) transferred onto a structured COC film. In TM, topography and phase images ( $A$ and $B$, respectively) have no hint of where the voids are. On the other hand, the UFM image (D) clearly shows the regions suspended and not visible in topography (C). The darker the UFM contrast, the larger the indentation. (E) The topography and UFM profiles of the lines indicated in (C) and (D). 

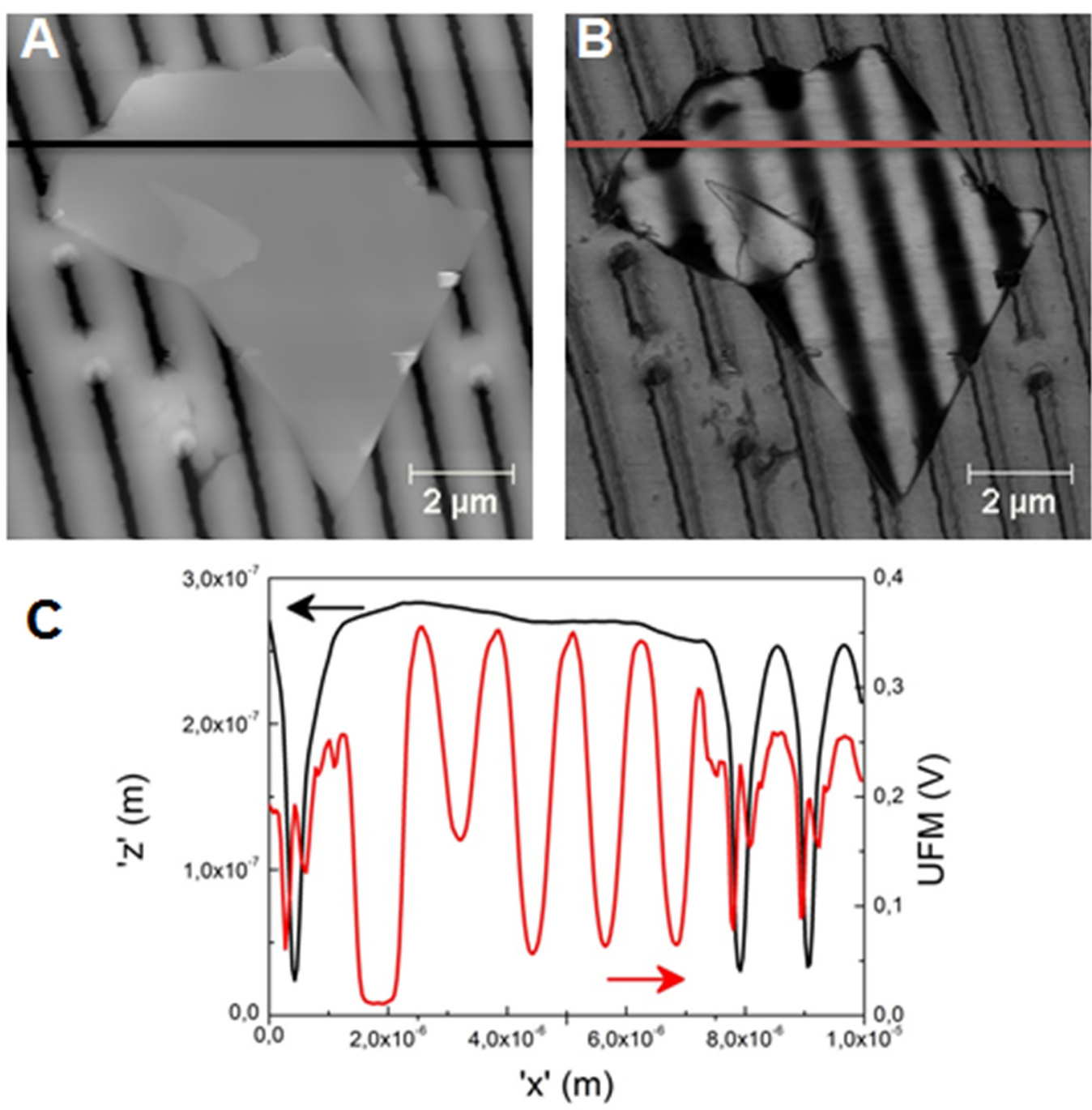

Figure 3: (A) The topography image of a $\mathrm{MoS}_{2}$ flake (around 15nm thick, excluding folded areas) placed on a ridge-structured COC film. (B) The UFM image shows the areas suspended in dark. (C) The topography and UFM profiles of the lines indicated in (A) and (B). 

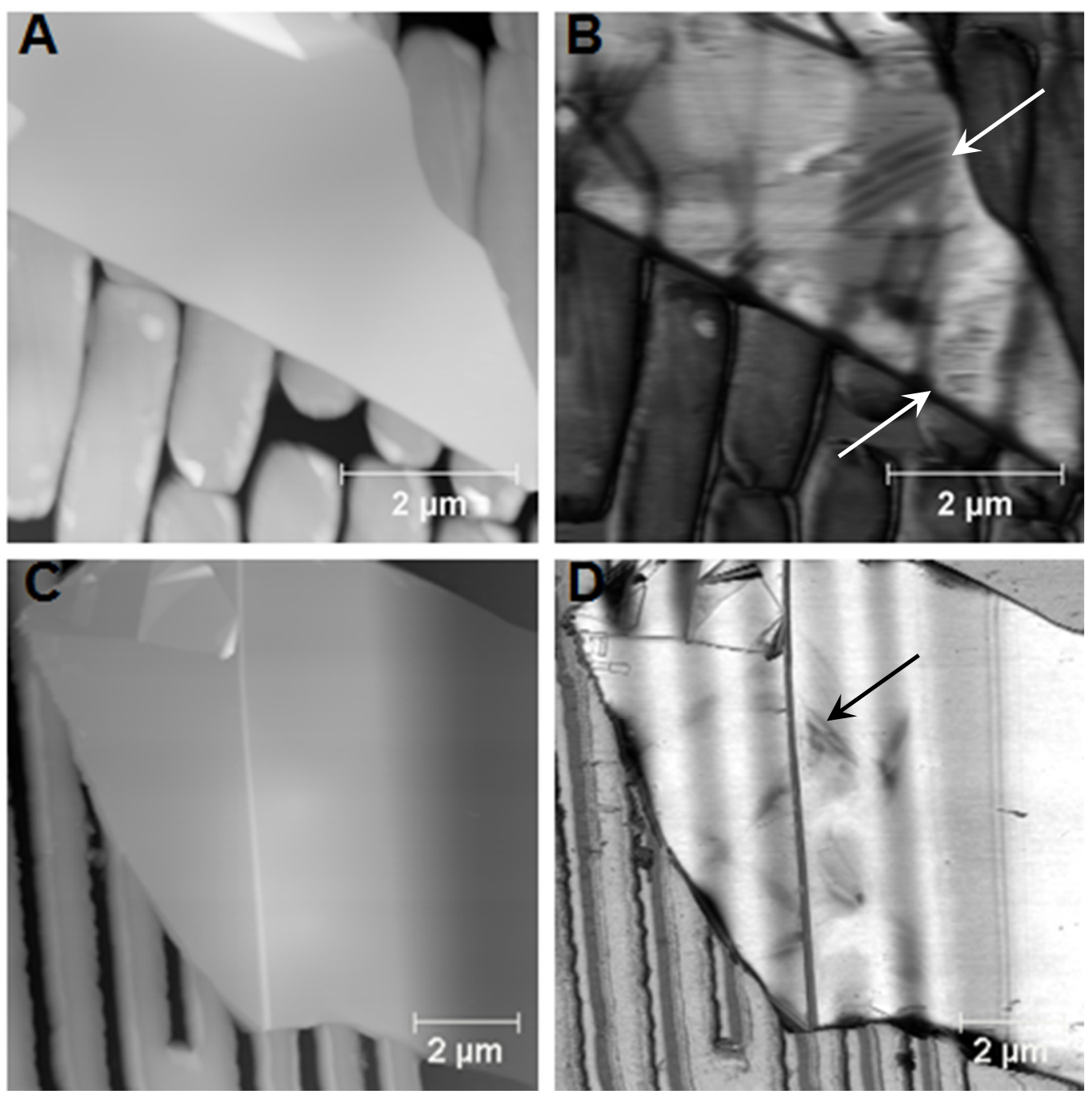

Figure 4: (A) Topography image of a graphite flake (around 50nm thick, excluding folded areas) on a randomly structured $\mathrm{COC}$ film. (B) In addition to the suspended regions, the UFM image clearly show that the internal contrast is quite rich. There are parallel lines probably due to delamination between adjacent graphene layers (upper white arrow) and irregular features probably due to surface damage (lower white arrow). (C) Topography image of another graphite flake (around 30nm thick, excluding folded areas) on the patterned COC substrate. (D) The UFM image shows, in addition to the suspended areas, some dark non-parallel lines only in correspondence of the suspended parts that might be due to some buckling induced by the pressure exerted through the tip (black arrow). 

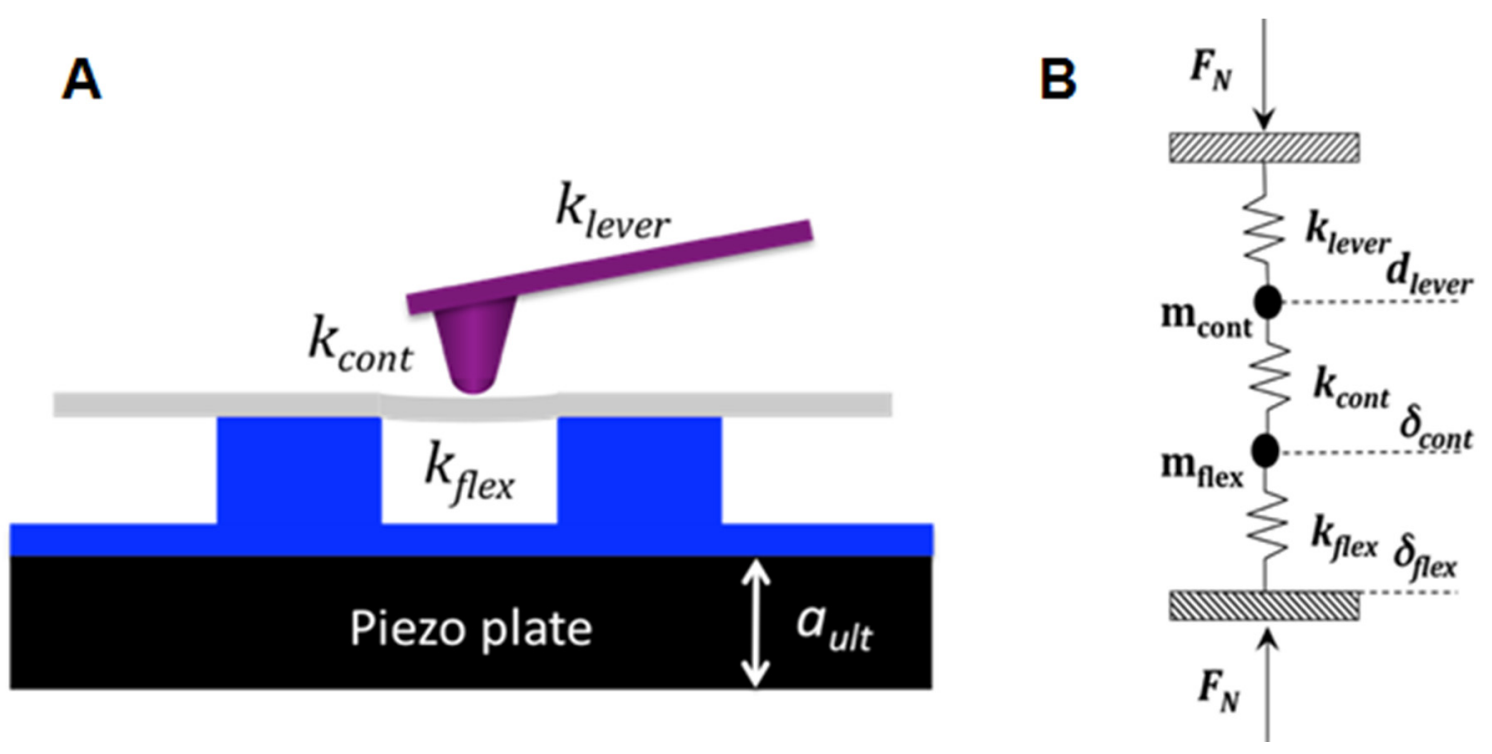

Figure 5: (A) A schematic drawing of a tip in contact with a suspended region of the platelet (in gray) where bending can occur. The indentation can be larger than on areas supported by the COC substrate (in blue), thus it needs a higher ultrasonic amplitude in order to break the contact and observe the non-linearity of the $F_{N}$ versus $\delta$ curves. (B) The two-spring model in Figure $1 \mathrm{C}$ can be extended to three with the introduction of the platelet bending $\delta_{\text {flex. }}$ In principle, the model can be further extended to four or more springs, to take into account internal defects such as a delamination or else. 


\section{References}

${ }_{1}^{1}$ Binnig G, Rohrer H, Gerber C and Weibel E 1982 Appl. Phys. Lett.40 178

${ }^{2}$ Binnig G, Quate CF and Gerber C 1986 Phys. Rev. Lett. 56930

3 Bhushan B 2016 Scanning Probe Microscopy in Nanoscience and Nanotechnology Springer Berlin ISBN-10: 3662502208

${ }^{4}$ Fuchs H and Bhushan B 2016 Biosystems Investigated by Scanning Probe Microscopy Springer Berlin Heidelberg ISBN-10: 3662519194

${ }^{5}$ Pingue P 2011 Scanning probe based nanolithography and nanomanipulation on graphene Chapter in the book Tseng AA Tip-based Nanofabrication: Fundamentals and Applications Springer Springer-Verlag ISBN-10: 1

${ }^{6}$ Glembocki OJ, Snow ES, Marrian CRK, Prokes SM and Katzer DS 1992 Ultramicroscopy 42-44 764

7 Schmid M, Crampin S and Varga P 2000 J. Elect. Spectr. 10971

${ }^{8}$ Flores M, Cisternas E, Correa JD and Vargas P 2013 Chem. Phys. 42349

${ }^{9}$ Zhao M, Gu X, Lowther SE, Park C, Jean YC and Nguyen T 2010 Nanotechnology 21225702

10 Yamada F and Kamiya I 2013 Appl. Surf. Sci. 271131

${ }^{11}$ Kay ND, Robinson BJ, Fal'ko VI, Novoselov KS and Kolosov OV 2014 Nano Lett. 143400

12 Nonnemacher M and Wickramasinghe HK 1992 Appl. Phys. Lett. 61168

${ }^{13}$ David L, Gomes S and Raynaud M 2007 J. Phys. D - Appl. Phys. 404337

14 Tovee PD and Kolosov OV 2013 Nanotechnology 241174

${ }^{15}$ Kolosov OV and Yamanaka K 1993 Japan J. of Appl. Phys. Part 2-Letters 32 L1095

${ }^{16}$ Burnham NA, Gremaud G, Kulik AJ, Gallo P-J and Ouveley F 1996 J. Vac. Sci. Technol. B 141308

${ }^{17}$ Chilla E, Rohrbeck W, Frohlich H-J, Koch R and Rieder KH 1992 Appl. Phys. Lett. 613107

${ }^{18}$ Rabe U and Arnold W 1994 App. Phys. Lett. 641423

${ }^{19}$ Burnham NA, Kulik AJ, Gremaud G, Gallo P-J and Ouveley F 1996 J. Vac. Sci. Technol. B 14794

${ }^{20}$ Cuberes MT, Assender HE, Briggs GAD and Kolosov OV 2000 J. Phys. D 332347

${ }^{21}$ Zhong Q, Innis D, Kjoller K and Elings VB 1993 Surf. Sci. Lett. 290 L688

22 Garcia R and Heruzo ET 2012 Nat. Nanotechnology 7217

${ }^{23}$ Ebeling D, Eslami B and De Jesus Solares S 2013 ACS Nano 1110387

24 Dinelli F, Biswas SK, Briggs GAD and Kolosov OV 1997 Appl. Phys. Lett. 711177

${ }^{25}$ Yamanaka K, Ogiso H and Kolosov OV 1994 Appl. Phys. Lett. 64178

${ }^{26}$ Yamanaka K, Kobari K and Tsuji T 2008 Jap. J. Appl. Phys. 476070

${ }^{27}$ Checanov AS, Low TS, Alli S, Kolosov OV and Briggs GAD 1996 IEEE Trans. Magn. 323696

${ }^{28}$ Dinelli F, Castell MR, Ritchie DA, Mason NJ, Briggs GAD and Kolosov OV 2000 Phil. Mag. A 802299

${ }^{29}$ Shekhawat $\mathrm{G}$ and Dravid VP 2005 Science 31089

30 Sarioglu AF, Atalar A and Degertekin FL 2004 Appl. Phys. Lett. 845368

${ }^{31}$ Verbiest GJ, Simon JN, Oosterkamp TH and Rost MJ 2013 Nanotechnology 23145704

${ }^{32}$ Kimura K, Kobayashi K, Matsushinge K and Yamada H 2013 Ultramicroscopy 13341

${ }^{33}$ Novoselov KS, Jiang D, Schedin F, Booth TJ, Khotkevich VV, Morozov SV and Geim AK 2005 PNAS 102 10451

${ }^{34}$ Pittenger B, Erina N and Su C 2012 Bruker Application note AN128, Rev. B0

${ }^{35}$ Goler S, Piazza V, Roddaro S, Pellegrini V and Beltram F 2011 J. Appl. Phys. 110064308 
${ }^{36}$ Bosse JL, Tovee PD, Huey BD and Kolosov OV 2014 J. Appl. Phys. 115144304

${ }^{37}$ Briggs GAD and Kolosov OV 2009 Acoustic Microscopy Oxford University Press

${ }^{38}$ Dinelli F, Biswas SK, Briggs GAD and Kolosov OV 2000 Phys. Rev. B 6113995

39 Derjaguin BV, Muller VM and Toropov YuP 1975 J. Colloid. Interface Sci. 53314

40 Johnson K 1994 Contact Mechanics Cambridge University Press

${ }^{41}$ Ventsel E and Krauthammer T 2001 Thin Plates Theory Marcel Dekker Inc. New York - Basel

42 https://www.entegris.com/resources/assets/6205-7329-0513.pdf

${ }^{43}$ Wang T, Ma C, Hu W, Chen Y and Chu J 2016 Micr. Res. Tech. 222470

${ }^{44}$ Castellanos-Gomez A, Buscema M, Molenaar R, Singh V, Janssen L, van der Zant HSJ and Steele GA 2014 2D Materials 1011002

45 Tsoukleri G, Parthenios J, Papagelis K, Jalil R, Ferrari AC, Geim AK, Novoselov KS and Galiotis C 2009 Small 52397

46 Jiang T, Huang R and Zhu Y 2014 Adv. Funct. Mat. 24396 